\title{
Target Feasibility Achievement in Enterprise-Driven Hierarchical Multidisciplinary Design
}

\author{
Harrison M. Kim* Deepak K. D. Kumar ${ }^{\dagger}$ Wei Chen ${ }^{\ddagger}$ \\ Department of Mechanical Engineering, Northwestern University, Evanston, IL, 60208, USA \\ Panos Y. Papalambros $\$$ \\ Department of Mechanical Engineering, University of Michigan, Ann Arbor, MI, 48109, USA
}

\begin{abstract}
Linking enterprise-wide business decision making with engineering decision making is demonstrated by integrating the enterprise utility optimization and the engineering product development approach under a multilevel optimization framework. The analytical target cascading process is used to set the right targets for the engineering design process that maximize utility at the enterprise level. A new methodology is proposed that systematically explores the engineering design space, often represented with a disconnected feasible domain. Analytical examples and an automotive suspension design case study are presented to demonstrate the methodology.
\end{abstract}

Key words: multidisciplinary design optimization, enterprise-driven design, analytical target cascading, discrete choice analysis, multinomial logit demand model, disconnected feasible domain.

\section{Introduction}

Designing a large-scale artifact involves multidisciplinary efforts in marketing, product development and production. There has been a number of efforts to integrate customer preference information in engineering design, e.g., Donndelinger and Cook, ${ }^{1}$ Besharati, et al.,${ }^{2,3} \mathrm{Li}$ and Azarm, ${ }^{4}$ Wassenaar and Chen, ${ }^{5}$ Cooper, et al., ${ }^{6} \mathrm{Gu}$, et al., ${ }^{7}$ Michalek, et al. ${ }^{8}$ They have demonstrated that a relationship exists between marketing and engineering in the enterprise. In this article the enterprise is defined as the organization that designs and produces an artifact to maximize its utility (e.g., profit). For simplicity, marketing and production are defined as the enterprise level and product development is defined as the engineering design.

Emphasis on enterprise-driven design models has led to incorporating demand and profit models that capture both producer and consumer needs. For example, Wassenaar and Chen ${ }^{5}$ developed demand models utilizing discrete choice analysis ${ }^{9}$ in a decision-based design (DBD) framework. ${ }^{5,7,10,11}$ This approach identifies the key customer attributes, i.e., product attributes that are of interest to customers. In Wassenaar's approach ${ }^{12,13}$ an optimal set of attribute targets is obtained at the enterprise level to maximize the net revenue of a firm. Considering engineering needs the socioeconomic and demographic background of customers, and time. The key customer attributes are assigned in the form of targets to be achieved in the engineering design process. To achieve the targets the engineering design efforts often involves multidisciplinary expertise and this design process can be realized by using multidisciplinary design optimization ${ }^{14,15}$ (MDO) approaches.

*Postdoctoral Research Fellow, Department of Mechanical Engineering, Northwestern University, Evanston, IL 60208, USA. e-mail: kimhm@northwestern.edu

${ }^{\dagger}$ Graduate student, Department of Mechanical Engineering, Northwestern University, Evanston, IL 60208, USA. e-mail: k-dileep@northwestern.edu

${ }^{\ddagger}$ Associate Professor, Department of Mechanical Engineering, Northwestern University, Evanston, IL 60208, USA, AIAA Associate Fellow. e-mail: weichen@northwestern.edu.

$\S$ Professor, Department of Mechanical Engineering, University of Michigan, MI 48109, USA, AIAA Senior Member. e-mail: pyp@umich.edu. 
In the original DBD framework proposed by Hazelrigg, ${ }^{10}$ the DBD approach is represented in a single optimization problem where decisions on product design and market strategies are made simultaneously. In practical implementations, it is unlikely to exercise a single optimization for a large, complex system. Also from the viewpoint of organizational infrastructure, decomposition and multilevel distributed approaches must be introduced. Figure 1 illustrates this decomposed hierarchical framework [see Cooper, et al., ${ }^{6}$ Michalek, et al. ${ }^{8}$. The enterprise-level product planning problem maximizes the utility $V$ with respect to the target performance $\mathbf{T}^{U}$ and the rest of the enterprise variables $\mathbf{x}_{e}$ subject to product development capability. At the engineering level, the objective is to minimize the deviation between the performance target and the response of the product $\mathbf{R}$ while satisfying the feasibility constraints $\mathbf{g}, \mathbf{h}$. An optimal set of attributes (i.e., targets) found at the enterprise level, however, may not lead to optimal engineering designs due to constraints introduced at the product development level. Thus engineering feasibility enforces adjustment of the attributes at the enterprise level. This adjustment may shift the enterprise utility value from its original optimal value. In return, however, a consistent feasible design is obtained satisfying the engineering constraints. Hence it is critical to have a systematic decision making tool to ensure preference consistency throughout product planning at the enterprise level and product development at the engineering level.

To achieve the targets identified from product planing an-

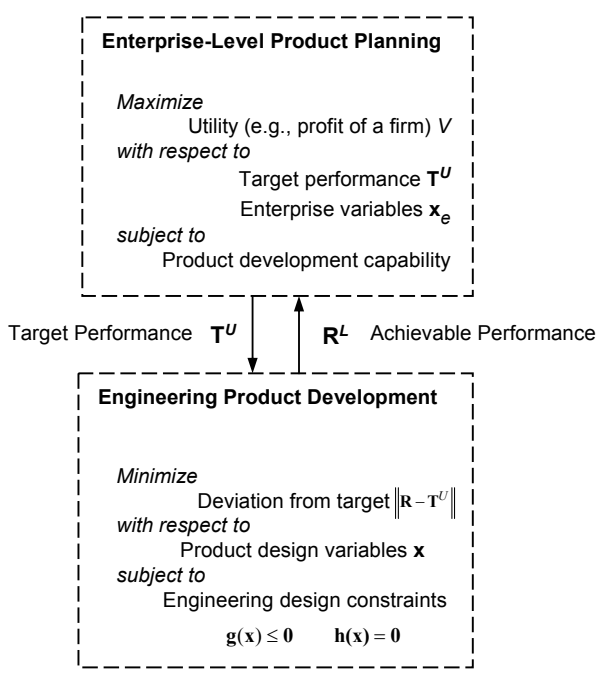

Figure 1. Interaction between enterprise product planning and product development. alytical target cascading (ATC) ${ }^{16-19}$ can be utilized for the product development process. ${ }^{6,8}$ ATC is a multilevel, multidisciplinary design methodology to find an optimal system design ensuring consistency among subsystems or disciplines and achieving the overall product targets assigned at the top of the hierarchy. In the field of MDO, several design architectures have been developed to support collaborative multidisciplinary design environment using distributed design optimization, e.g., concurrent subspace optimization $(\mathrm{CSSO})^{20}$ and collaborative optimization $(\mathrm{CO}) .{ }^{21} \mathrm{~A}$ comprehensive review of the multidisciplinary design optimization architectures is provided by Kroo. ${ }^{15}$

The key difference between ATC and most of the previous MDO formulations, including $\mathrm{CO}$, is that, with ATC, the original problem is decomposed hierarchically at multiple levels, while the interconnections between the multiple subsystems at each level are considered and coordinated at one level above. The ATC problem $P_{i j}$ associated with the $j^{\text {th }}$ element at the $i^{\text {th }}$ level of the hierarchy is formulated in Eq. (1). Responses $\hat{\mathbf{R}}_{i j}=\left[\tilde{\mathbf{R}}_{i j}, \mathbf{R}_{i j}\right]^{T}=$ $\mathbf{r}_{i j}\left(\mathbf{R}_{(i+1) k_{1}}, \ldots, \mathbf{R}_{(i+1) k_{c_{i j}}}, \mathbf{x}_{i j}, \mathbf{y}_{i j}\right)$ are computed by means of analysis and/or simulation models. The vector of all optimization variables is $\hat{\mathbf{x}}=\left[\mathbf{x}_{i j}, \mathbf{y}_{i j}, \mathbf{y}_{(i+1) k_{1}}, \ldots, \mathbf{y}_{(i+1) k_{c_{i j}}}, \mathbf{R}_{(i+1) k_{1}}, \ldots, \mathbf{R}_{(i+1) k_{c_{i j}}}, \epsilon_{i j}^{\mathbf{R}}, \epsilon_{i j}^{\mathbf{y}}\right]^{T}$ and $\mathbf{x}_{i j}$ is the vector of local design variables. $\tilde{\mathbf{R}}_{i j}$ corresponds to responses linked to local targets and $\mathbf{R}_{i j}$ corresponds to responses linked to cascaded targets. $\epsilon_{i j}^{\mathbf{R}}$ and $\epsilon_{i j}^{\mathbf{y}}$ are the tolerance optimization variables for ensuring consistency. Superscripts $(\cdot)^{U}$ and $(\cdot)^{L}$ denote values passed down and up from the upper and lower levels, respectively. The vector $\mathbf{T}$ denotes local targets. $\mathbf{g}_{i j}$ and $\mathbf{h}_{i j}$ are local design constraints. Weights $\mathbf{w}$ are assigned to the deviation terms in the objective.

$$
\begin{aligned}
& P_{i j}: \min _{\mathbf{x}_{i j}} \quad \mathbf{w}_{i j}^{\tilde{\mathbf{R}}}\left\|\tilde{\mathbf{R}}-\mathbf{T}_{i j}\right\|+\mathbf{w}_{i j}^{\mathbf{R}}\left\|\mathbf{R}_{i j}-\mathbf{R}_{i j}^{U}\right\|+\mathbf{w}_{i j}^{\mathbf{y}}\left\|\mathbf{y}-\mathbf{y}_{i j}^{U}\right\|+\epsilon_{i j}^{\mathbf{R}}+\epsilon_{i j}^{\mathbf{y}} \\
& \text { subject to } \\
& \sum_{k \in C_{i j}}\left\|\mathbf{R}_{(i+1) k}-\mathbf{R}_{(i+1) k}^{L}\right\| \leq \epsilon_{i j}^{\mathbf{R}} \quad \sum_{k \in C_{i j}}\left\|\mathbf{y}_{(i+1) k}-\mathbf{y}_{(i+1) k}^{L}\right\| \leq \epsilon_{i j}^{\mathbf{y}} \\
& \mathbf{g}_{i j}\left(\hat{\mathbf{R}}_{i j}, \mathbf{x}_{i j}, \mathbf{y}_{i j}\right) \leq \mathbf{0} \quad \mathbf{h}_{i j}\left(\hat{\mathbf{R}}_{i j}, \mathbf{x}_{i j}, \mathbf{y}_{i j}\right)=\mathbf{0}
\end{aligned}
$$

Most of the other work considered up to this point (e.g., $\mathrm{CO}^{21}$ and BLISS ${ }^{22}$ ) has been concerned with decomposing a problem into a series of problems, all at one non-hierarchical level, but then solving the problem using bi-level optimization formulations. ATC operates by formulating and solving a minimum 
deviation optimization problem for each element in the hierarchy. Compared to the existing bi-level optimization formulations, the multilevel hierarchical modeling facilitated by the ATC approach better matches a multi-layered organizational decision making infrastructure, where subsystems and components can be supplied by different organizational units or outsourced to independent companies.

At the initial stage of the product development process, the targets for the engineering design are set by maximizing the utility at the enterprise level that reflects preferences of customers/producers. The initial (utopia) targets are not usually perfectly achievable at the engineering level. On the other hand, the best available feasible design (i.e., with the minimum deviation from the targets) at the engineering level may not correspond to the best utility at the enterprise level. An alternative feasible design at the engineering level with a higher deviation (i.e., less favored alternative) from the utopia targets may correspond to a better utility at the enterprise level. The proposed approach offers a systematic way to match the design utility at both product planning and product development process. It allows exploration in the engineering design space, often represented with a disconnected feasible domain, and guides the solution process to a better design in the enterprise context.

The problem formulation and the solution algorithm are proposed in the following sections. The proposed approach is demonstrated using three examples: an analytical example with a single optimum in the utility, an analytical example with multiple local optima in the utility and a vehicle suspension design example that includes customer preference choices.

\section{Enterprise Model}

The enterprise level objective is to maximize a utility, such as the profit of a firm. As shown in Figure 2,

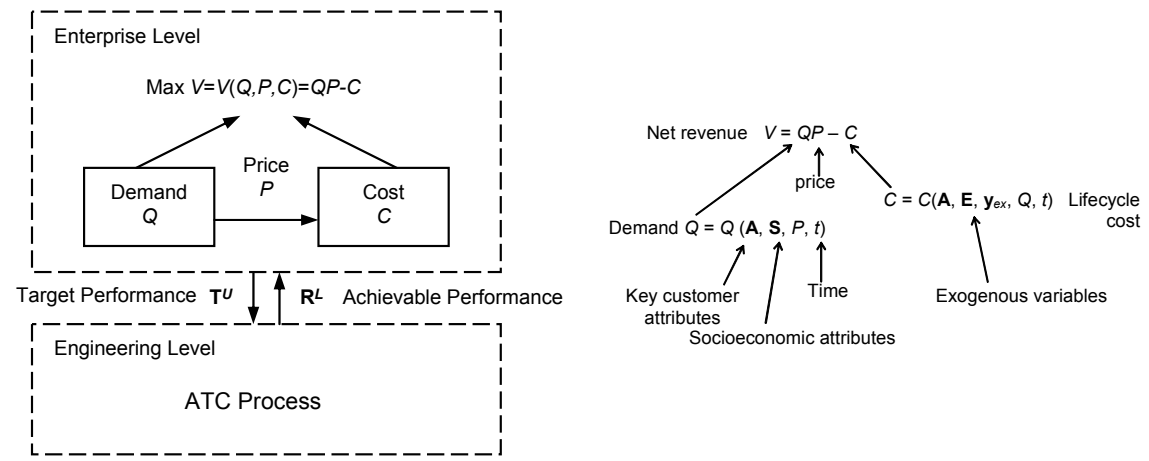

Figure 2. Bi-level enterprise decision flow and net revenue model.

the demand $Q$ plays a critical role in assessing both revenue and expenditure, and ultimately the profit (i.e., net revenue $V$ ). To use the enterprise model to guide engineering product development, $Q$ is expressed here as a function of the key customer attributes $\mathbf{A}$, socioeconomic attributes $\mathbf{S}$ of the market population, price $P$ and time $t$; the expenditure $C$ (i.e., life cycle cost including manufacturing cost and others) is a function of the customer attributes $\mathbf{A}$, the engineering attributes $\mathbf{E}$, exogenous variables $\mathbf{y}_{e x}$, demand $Q$ and time $t$. The key customer attributes $\mathbf{A}$ are product features (next to brand, price, and warranty) that a customer typically considers when purchasing the product and the engineering attributes $\mathbf{E}$ are product properties that are of interest only to design engineers. Targets for the ATC process $\mathbf{T}$ are the targets for both A and $\mathbf{E}$. To assist engineering decision making, the relationships of $\mathbf{A}$ and $\mathbf{E}$ with design options are established through engineering analyses.

Demand modeling using Discrete Choice Analysis (DCA) and Conjoint Analysis (CA) have been widely used in the marketing and transportation communities [Green and Srinivasan, ${ }^{23}$ Ben-Akiva and Lerman, ${ }^{9}$ Koppelman and Sethi ${ }^{24}$ ]. Researchers in the design community [Besharati, et al., ${ }^{3} \mathrm{Li}$ and Azarm, ${ }^{4}$ Wassenaar and Chen, ${ }^{5}$ Michalek, et al. ${ }^{8}$ have also had success in integrating such models in their work. In this article the DCA approach is adopted for demand modeling and the multinomial logit (MNL) model is used because it has a closed form and the Gumbel ${ }^{9}$ error distribution closely approximates the normal distribution.

The choice probability MNL model is shown in Eq. (2), where $\operatorname{Pr}_{n}(i)$ is the probability that respondent $n$ 
chooses alternative $i, J$ is the choice set that is available to individual $n$, and $W$ is the observable/deterministic part of the utility function:

$$
\operatorname{Pr}_{n}(i)=\frac{e^{W_{i n}}}{\sum_{j \in J} e^{W_{j n}}}
$$

This formulation implies that the probability of choosing an alternative increases monotonically with an increase in systematic or deterministic utility of that alternative and decreases with the increase of systematic utility of any or all of the other alternatives. Also, this form implies that when one is choosing between two alternatives, all other alternatives are irrelevant. This offers some computational convenience since it allows for the addition/removal of an alternative to/from the choice set without affecting the structure or parameters of the model.

\section{Problem Formulation and Solution Algorithm}

The original unconstrained enterprise level utility optimization problem is

$$
P_{e n t}^{0}: \max _{\mathbf{T}, \mathbf{x}_{e}} U\left(\mathbf{T}, \mathbf{x}_{e}\right)
$$

where the objective is to maximize the utility $U$ that is a function of targets $\mathbf{T}$ for the engineering problem and of other enterprise variables $\mathbf{x}_{e}$. After solving Eq.(3), the utopia target $\mathbf{T}^{*}$ that maximizes the utility $U$ is assigned to the engineering problem. Then the engineering problem finds an optimal response $\mathbf{R}^{L 0}$ to the utopia target with the minimum deviations (see Figure 1).

In most engineering design cases, it is uncommon to meet the utopia target perfectly due to the trade-off nature of the multiple target values or physical feasibility (i.e., no feasible design is available to meet the targets perfectly). Based on the engineering response $\mathbf{R}^{L 0}$ the utility is adjusted with the corresponding values for $\mathbf{T}$ and $\mathbf{x}_{e}$.

If the feasible domain in the engineering problem is disconnected, the engineering design with the minimum deviation from the targets may not correspond to the maximum utility value. Figure 3 illustrates one-dimensional and two-dimensional cases where the minimum deviation from the utopia target does not match the best available utility. Points A and B are both engineering local optima with the minimum deviation from the target. The deviation for the point $\mathrm{A}$ is smaller, but the corresponding utility is not higher than that of point B.

The proposed algorithm leads the engineering design to match the enterprise-driven preferences (i.e., targets) by systematically exploring a multiply-connected feasible design space at the engineering level. The final design is consistent with the targets and improved in the enterprise utility sense. To enable the move from one feasible domain to another, an additional constraint $C_{a u x}$ is imposed in the enterprise problem based on the engineering response $\mathbf{R}^{L 0}$ as shown in Eq. (4). The physical meaning of the additional constraint is that it imposes a minimum geometric distance from the utopia target so that the enterprise problem is forced to find another target for the engineering problem.

$$
C_{\text {aux }}:\left\|\mathbf{T}-\mathbf{R}^{L 0}\right\| \geq \Delta=\left\|\mathbf{T}^{*}-\mathbf{R}^{L 0}\right\|
$$

For example, in Figure 4 the engineering problem returns point $\mathrm{A}$ to the enterprise problem with the minimum deviation from the utopia target $\mathbf{T}^{*}$. A circular inequality constraint is imposed on the utopia target at the center, with the distance between the utopia target and the engineering response as its radius. The modified enterprise problem $P_{\text {ent }}^{\prime}$ (Eq. 5) generates a new target $\mathbf{T}^{\prime}$ for the engineering problem (see Figure 4). Based on the new target the engineering problem finds point $\mathrm{B}$ as the optimum with the minimum deviation from the new target $\mathbf{T}^{\prime}$. Point B is farther from the original utopia target, however the corresponding utility is higher than that of point A. As a result, point B is selected as the optimal engineering design that has a better utility value.

$$
\begin{aligned}
P_{\text {ent }}^{\prime}: & \max _{\mathbf{T}, \mathbf{x}_{e}} U\left(\mathbf{T}, \mathbf{x}_{e}\right) \\
& \text { subject to } \\
& C_{\text {aux }}:\left\|\mathbf{T}-\mathbf{R}^{L 0}\right\| \geq \Delta=\left\|\mathbf{T}^{*}-\mathbf{R}^{L 0}\right\|
\end{aligned}
$$




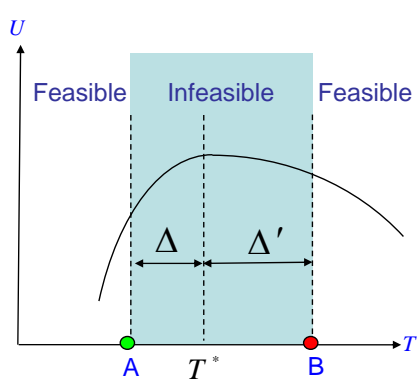

(a) One-dimensional case

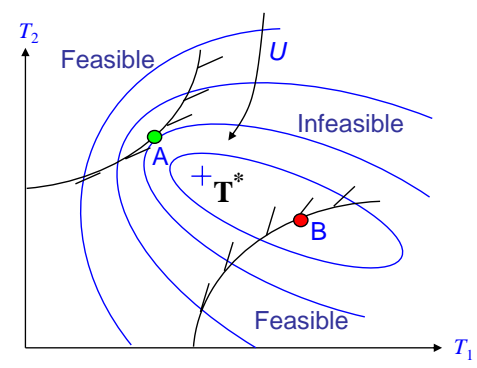

(b) Two-dimensional case

Figure 3. Utilities with engineering feasible domain imposed. Points A and B are both engineering local optima with the minimum deviation from the target. The deviation for the point $\mathrm{A}$ is smaller, but the corresponding utility is not higher than that of point $B$.

After solving the modified enterprise problem Eq. (5), however, the new target for the engineering problem may not necessarily lead to another disconnected feasible domain. To visualize this situation in Figure 5 a unimodal utility function is plotted with the engineering feasible domain overlapped. The shaded region denotes the infeasible engineering domain, while there exist disconnected feasible domain on both sides of the infeasible domain. At the first iteration the engineering problem returns a response $\left(T^{*}-\Delta\right)$. A constraint as shown in Eq. (4) is added to the enterprise problem and the new target is assigned at $\left(T^{*}+\Delta\right)$. The new target $\left(T^{*}+\Delta\right)$ does not force the engineering problem to find the minimum deviation from the target $R^{\prime}$ that is at the boundary of the other feasible domain because the right feasible domain is farther than the original left engineering feasible domain. Thus the engineering design returns to the previous response at $\left(T^{*}-\Delta\right)$. To avoid returning to the previous solution, additional slope information is utilized to adjust the radius of the restricted feasible domain in the enterprise problem.

After finding the minimum deviation engineering design at $\left(T^{*}-\Delta\right)$, the gradient of the utility function $\alpha$ is obtained. When the next target for the engineering problem $\left(T^{*}+\Delta\right)$ is obtained by solving the modified enterprise problem, the gradient of the utility function $\phi$ is also obtained. If the engineering problem returns the same response $\left(T^{*}-\Delta\right)$, then the constraint radius is increased up to $\Delta^{\prime}$ with the consideration of the gradient ratio:

$$
\Delta^{\prime} \approx \frac{\alpha}{\phi} \Delta
$$

An updated target $\left(T^{*}+\gamma \Delta^{\prime}\right)$ is assigned to the engineering problem and the engineering problem returns $R^{\prime}$ as response, where $\gamma$ is the step size for updating the new radius of the constraint that takes a value between 0 and 1 .

Hence the enterprise problem repeats assigning a new target if the engineering problem returns the same response as far as the utility improvement is expected. Eq. (6) provides the upper limit for increasing the radius of the constraint as it is based on a linear approximation of the utility function as shown in Figure

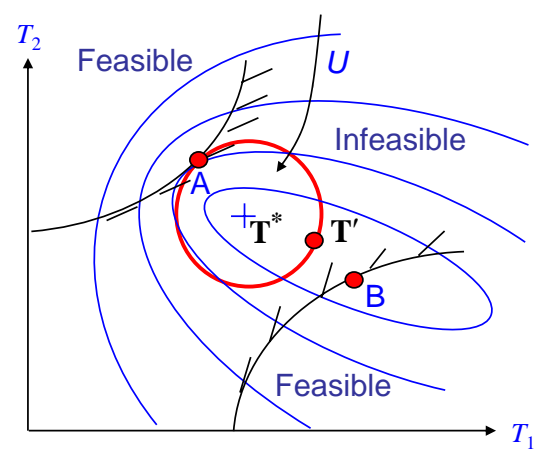

Figure 4. A circular constraint imposes a new target $\mathbf{T}^{\prime}$ for the engineering problem.

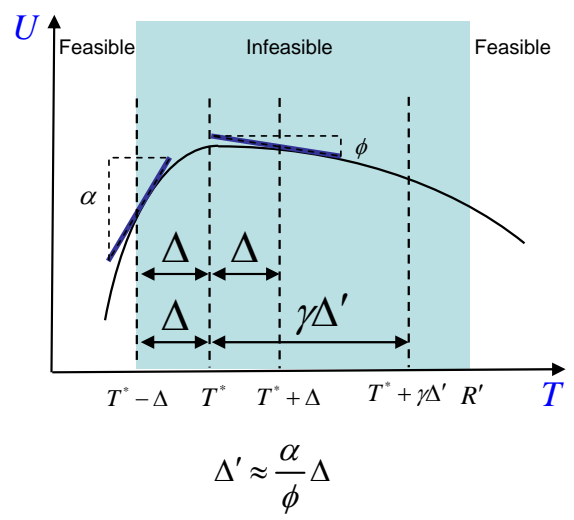

Figure 5. Updating the radius $\Delta$ in the additional constraint in the modified enterprise problem: the slope is considered to set the radius of the constraint (Eq.(4)) 
5.

As soon as an engineering level design is found with a better utility, the solution process stops, i.e., the goal of the solution algorithm is to find a feasible engineering design with a better utility. The proposed algorithm does not attempt to find the global optimum, rather it explores the engineering feasible domain to find alternative feasible design with a better utility if it exists in a disconnected feasible domain. It is also assumed that the engineering problem always finds the best solution, i.e., a feasible design with the minimum deviation from the target either by local or global search algorithm. The proposed algorithm is summarized as Algorithm III.1 (see also Figure 6).

\section{Algorithm III.1}

Start with $\mathbf{x}_{0}$.

Solve the original enterprise problem $\left(P_{\text {ent }}^{0}\right)$ and find the utopia target $\mathbf{T}^{*}$.

Solve the engineering problem $\left(P_{\text {eng }}\right)$ by using the ATC and obtain the response $\mathbf{R}^{L 0}$ with the minimum deviation from

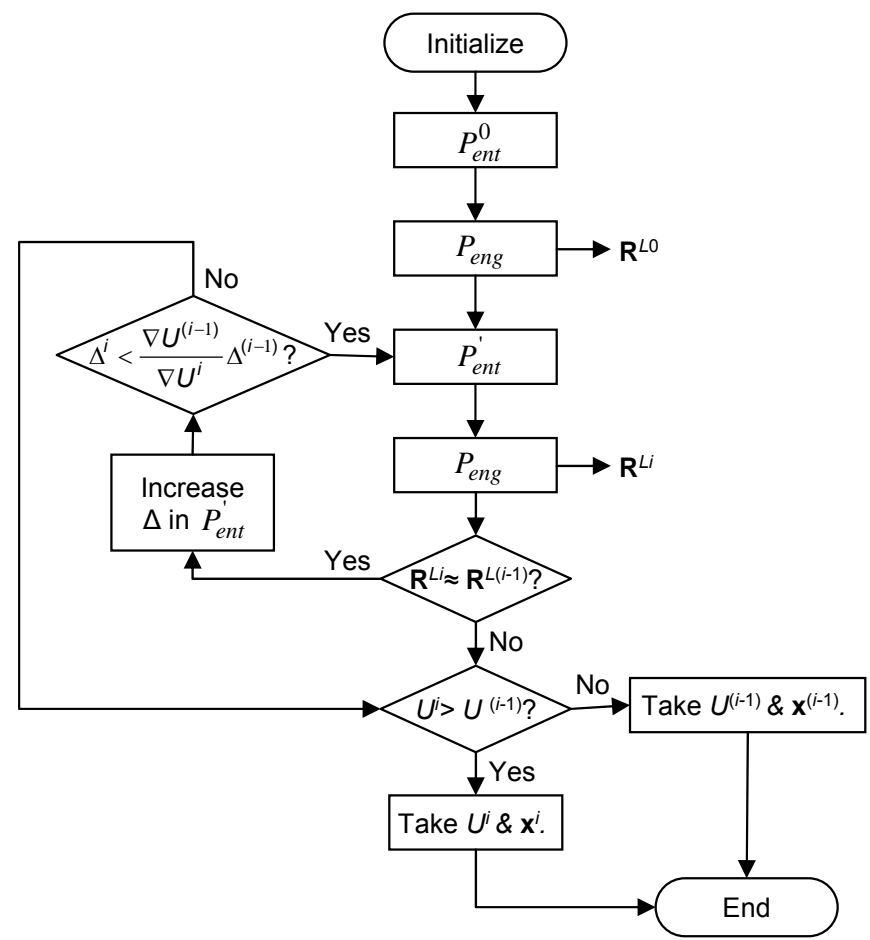
the target $\mathbf{T}^{*}$.

Add an additional constraint $C_{a u x}$ in the enterprise problem and find a new target $\mathbf{T}^{i}$ by solving the modified enterprise problem $\left(P_{\text {ent }}^{\prime}\right)$.

Figure 6. Solution algorithm

Solve the engineering problem and obtain the response $\mathbf{R}^{L i}$ with the minimum deviation from the target $\mathbf{T}^{i}$.

If $\mathbf{R}^{L i} \approx \mathbf{R}^{L(i-1)}$, increase $\Delta$ in $C_{a u x}$ in $P_{\text {ent }}^{\prime}$ and solve $P_{\text {ent }}^{\prime}$ and the engineering problems again.

Repeat until convergence.

End.

\section{Demonstration}

Three examples are presented to demonstrate the above ideas. Two analytical examples show progress to a better enterprise level design while successfully exploring a disconnected feasible engineering domain. The first one has a single utility optimum and the second has multiple local utility optima at the enterprise level while the engineering feasible domain is disconnected. The lower level problems are decomposed at multiple levels and are solved using ATC. A vehicle design problem involving suspension system design is also studied for enterprise profit maximization utilizing real customer purchase data for the demand model along with market share behavior with demographic information.

\section{A. Analytical Examples}

1. Utility with Single Optimum

An analytical geometric programming problem ${ }^{17}$ is modified to demonstrate the proposed algorithm. Top enterprise level problem is denoted as the upper level problem and the bottom level product development 
problem is denoted as the lower level problem. Targets $T_{1}, T_{2}$ are assigned to the lower level problem Eq. (8) after solving the upper level problem Eq. (7).

$$
\begin{gathered}
P_{\text {ent }}^{0}: \min _{T_{1}, T_{2}} \quad-U=\sqrt{\left(T_{1}-2\right)^{2}+\left(4 T_{2}-1\right)^{2}}+\sqrt{\left(T_{1}-2\right)^{2}+\left(4 T_{2}+1\right)^{2}} \\
\min _{x_{1}, x_{2}, \ldots, x_{14}}\left(x_{1}-T_{1}\right)^{2}+\left(x_{2}-T_{2}\right)^{2} \\
\text { subject to } \\
g_{1}:\left(x_{3}^{-2}+x_{4}^{2}\right) / x_{5}^{2} \leq 1 \quad g_{2}:\left(x_{5}^{2}+x_{6}^{-2}\right) / x_{7}^{2} \leq 1 \\
g_{3}:\left(x_{8}^{2}+x_{9}^{2}\right) / x_{11}^{2} \leq 1 \quad g_{4}:\left(x_{8}^{-2}+x_{10}^{2}\right) / x_{11}^{2} \leq 1 \\
g_{5}:\left(x_{11}^{2}+x_{12}^{-2}\right) / x_{13}^{2} \leq 1 \quad g_{6}:\left(x_{11}^{2}+x_{12}^{2}\right) / x_{14}^{2} \leq 1 \\
g_{7}:-\left(0.1\left(x_{1}-3\right)^{2}+4.5-x_{2}\right)\left(-\left(x_{1}-16\right)^{2}+2-x_{2}\right) \leq 0 \\
h_{1}: x_{1}^{2}=x_{3}^{2}+x_{4}^{-2}+x_{5}^{2} \quad h_{2}: 10 x_{2}^{2}=x_{5}^{2}+x_{6}^{2}+x_{7}^{2} \\
h_{3}: x_{3}^{2}=x_{8}^{2}+x_{9}^{-2}+x_{10}^{-2}+x_{11}^{2} \quad h_{4}: x_{6}^{2}=x_{11}^{2}+x_{12}^{2}+x_{13}^{2}+x_{14}^{2} \\
-20 \leq x_{3}, x_{4}, \ldots, x_{14} \leq 20
\end{gathered}
$$

The lower level problem Eq. (8) is decomposed at two levels (see Figure 7) and solved by ATC. After the top level problem is solved, the targets $T_{1}, T_{2}$ are assigned to the lower level problem $P_{s}$. After solving $P_{s}$, the bottom level problems $P_{s u b 1}, P_{s u b 2}$ are solved based on the targets $x_{3}^{U}, x_{6}^{U}, x_{11}^{U}$ found in that $P_{s}$ problem. Note that $x_{11}$ is a linking variable, i.e., common design variable that is shared by $P_{s u b 1}$ and $P_{s u b 2}$. After the iterative ATC process between $P_{s}$ and $P_{s u b 1}, P_{s u b 2}$ converges, the overall response with respect to the targets $T_{1}, T_{2}$ is passed up to the enterprise level problem and the utility is adjusted completing one iteration in Table 1.

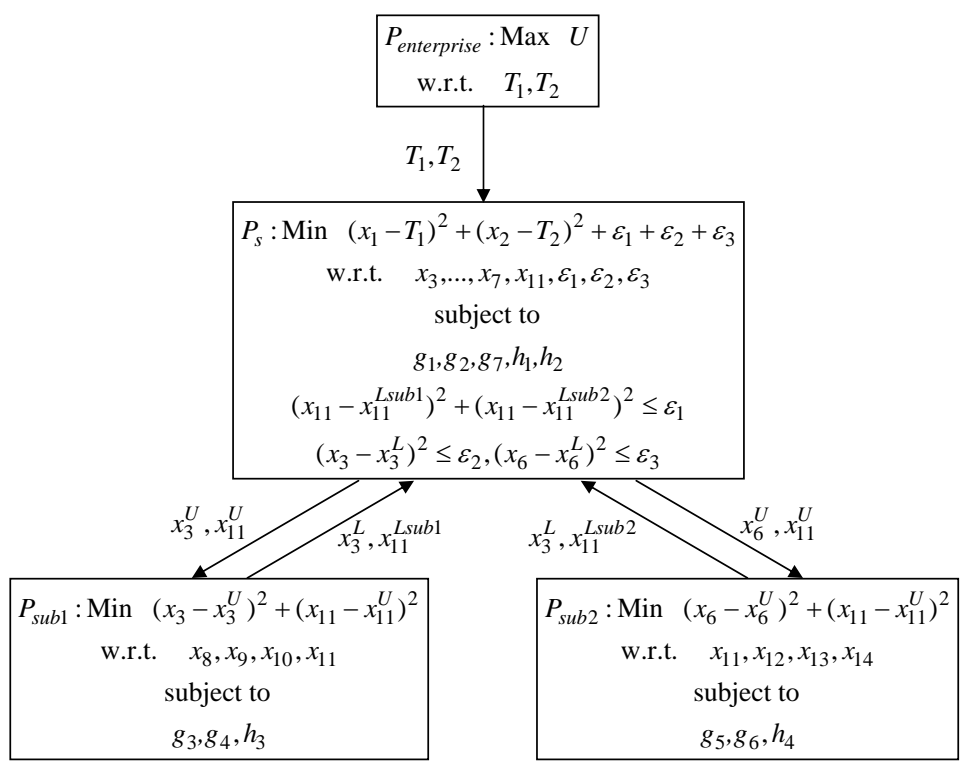

Figure 7. Decomposed problem hierarchy following analytical target cascading.

Note that the lower level problem Eq. (8) has disconnected feasible domain with respect to $T_{1}, T_{2}$. The disconnected feasible domain is plotted over the utility domain in Figure 8. For the unconstrained upper level problem, the optimal target, i.e., utopia target, is found at $\mathbf{T}^{*}=(2.0,0.079)$. Based on the utopia target, the lower level problem finds an optimal response with the minimum deviation at $\mathbf{R}^{L 0}=(4.006,4.601)$. Based on the lower level response $\mathbf{R}^{L 0}$, a constraint in Eq. (4) is added to the upper level problem and the upper level problem is solved again to assign a new target $\mathbf{T}^{\prime}$ to the lower level problem. An updated lower level solution $\mathbf{R}^{L 1}$ is found near the previous solution $\mathbf{R}^{L 0}$, i.e., the 


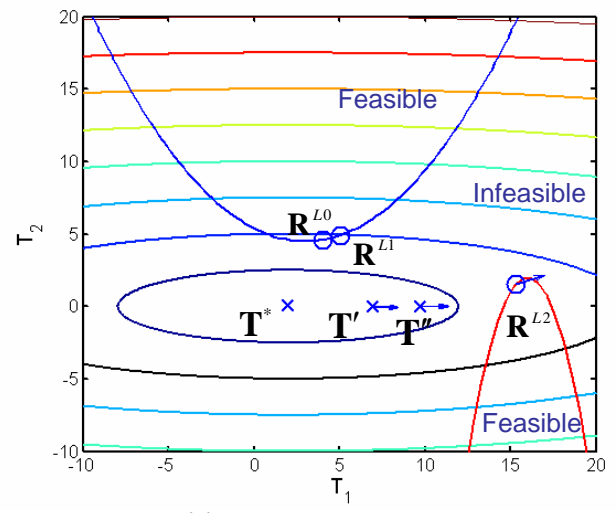

(a) Utility with single optimum

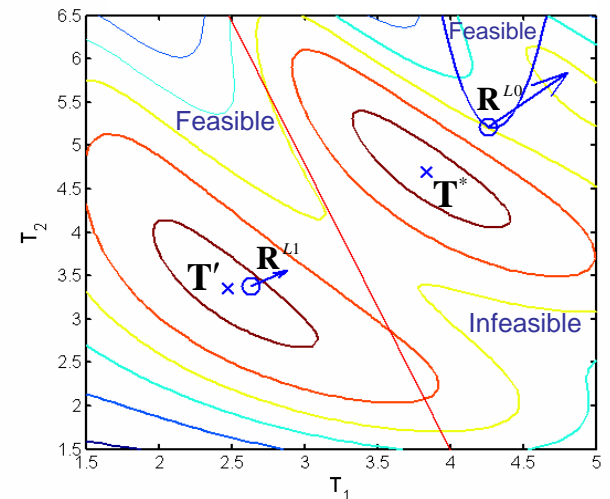

(b) Utility with multiple optima

Figure 8. Disconnected feasible domains are mapped over the utility space. The arrows represent the gradient.

lower level response is found in the same feasible domain. A newly assigned target $\mathbf{T}^{\prime \prime}$ based on the slope information (Eq. (6)) from the modified upper level problem guides the lower level problem to find the optimal response $\mathbf{R}^{L 2}$ in other feasible domain. The corresponding utility value for $\mathbf{R}^{L 2}$ is better and the lower level response is accepted as a solution. At the optimum, the solution is found at $\left(x_{3}, \ldots, x_{11}\right)=(15.30,1.50,5.09,-0.069,0.21,4.77,0.30,2.57,0.28,2.55,2.58,-0.84,2.84,2.71)$. The iteration process is summarized in Table 1 . Note that the responses are getting closer to the all-at-once (AAO) solution that is obtained when the decomposed problems in Eq. (7) and Eq. (8) are solved in a single integrated problem.

Table 1. Iteration History: Utility with Single Optimum

\begin{tabular}{r|c|c|c||c|c|c}
\hline Iteration & Target & Target & Desired & Response & Response & Actual \\
& $T_{1}$ & $T_{2}$ & utility & $R_{1}$ & $R_{2}$ & utility \\
\hline 1 & 2.000 & 0.079 & -2 & 4.006 & 4.601 & -37.029 \\
2 & 6.478 & -0.005 & -10.093 & 4.772 & 4.814 & -39.855 \\
3 & 9.372 & -0.005 & -15.610 & 14.177 & -1.323 & -29.243 \\
\hline AAO Solution & & & & 14.333 & -0.780 & -25.52 \\
\hline
\end{tabular}

\section{Utility with Multiple Local Optima}

The utility function at the upper level with multiple local maxima is defined as follows:

$$
P_{\text {ent } 0}: \max _{T_{1}, T_{2}} \quad U=2+0.01\left(T_{2}-T_{1}^{2}\right)^{2}+\left(4-T_{1}\right)^{2}+2\left(4-T_{2}\right)^{2}+7 \sin \left(0.55 T_{1}\right) \sin \left(0.6 T_{1} T_{2}\right) .
$$

After solving the problem in Eq. (9), targets $T_{1}, T_{2}$ are assigned for the lower level design problem:

$$
\begin{gathered}
\min _{x_{1}, x_{2}, \ldots, x_{14}}\left(x_{1}-T_{1}\right)^{2}+\left(x_{2}-T_{2}\right)^{2} \\
\text { subject to } \\
g_{1}:\left(x_{3}^{-2}+x_{4}^{2}\right) / x_{5}^{2} \leq 1 \quad g_{2}:\left(x_{5}^{2}+x_{6}^{-2}\right) / x_{7}^{2} \leq 1 \\
g_{3}:\left(x_{8}^{2}+x_{9}^{2}\right) / x_{11}^{2} \leq 1 \quad g_{4}:\left(x_{8}^{-2}+x_{10}^{2}\right) / x_{11}^{2} \leq 1 \\
g_{5}:\left(x_{11}^{2}+x_{12}^{-2}\right) / x_{13}^{2} \leq 1 \quad g_{6}:\left(x_{11}^{2}+x_{12}^{2}\right) / x_{14}^{2} \leq 1 \\
g_{7}:-\left(10\left(x_{1}-4.3\right)^{2}+5.2-x_{2}\right)\left(-3.3\left(x_{1}-4.15\right)+1-x_{2}\right) \leq 0 \\
h_{1}: x_{1}^{2}=x_{3}^{2}+x_{4}^{-2}+x_{5}^{2} \quad h_{2}: x_{2}^{2}=x_{5}^{2}+x_{6}^{2}+x_{7}^{2} \\
h_{3}: x_{3}^{2}=x_{8}^{2}+x_{9}^{-2}+x_{10}^{-2}+x_{11}^{2} \quad h_{4}: x_{6}^{2}=x_{11}^{2}+x_{12}^{2}+x_{13}^{2}+x_{14}^{2} \\
-20 \leq x_{3}, x_{4}, \ldots, x_{14} \leq 20 .
\end{gathered}
$$


The lower level problem is decomposed and solved as in the first example. The contour plot of the utility function with the overlapped lower level constraints is presented in Figure 8. For the unconstrained upper level problem, the optimal (utopia) target is found at $\mathbf{T}^{*}=(3.837,4.695)$. Based on this target, the lower level problem finds an optimal response with the minimum deviation at $\mathbf{R}^{L 0}=(4.259,5.216)$. Based on response $\mathbf{R}^{L 0}$ a constraint, as shown in Eq. (4), is added to the upper level problem and the upper level problem is solved again to assign a new target $\mathbf{T}^{\prime}$ to the lower level problem. The additional constraint guides the search process to find another local optimum in the utility space and assigns a new target for the lower level problem. The lower level problems finds an optimal solution $\mathbf{R}^{L 1}$ in the other region of the feasible domain. After checking that the corresponding utility value for $\mathbf{R}^{L 1}$ is better than the previous one, the lower level response is accepted as a solution. At the optimum, the solution is found at $\left(x_{3}, \ldots, x_{11}\right)=(2.63,3.38,2.19,0.94,1.05,3.03,1.10,0.92,-1.13,-0.96,1.45,0.85,1.87,1.68)$. The iteration process is summarized in Table 2 .

Table 2. Iteration History: Utility with Multiple Optima

\begin{tabular}{r|c|c|c||c|c|c}
\hline Iteration & Target & Target & Desired & Response & Response & Actual \\
& $T_{1}$ & $T_{2}$ & utility & $R_{1}$ & $R_{2}$ & utility \\
\hline 1 & 3.837 & 4.695 & 1.903 & 4.259 & 5.216 & -10.17 \\
2 & 2.470 & 3.356 & 1.365 & 2.634 & 3.383 & 0.846 \\
\hline AAO Solution & & & & 2.718 & -3.233 & 0.921 \\
\hline
\end{tabular}

These two analytical examples demonstrate that the proposed approach successfully explores the enterprise utility space to meet the enterprise objective with a consistent feasible engineering design.

\section{B. Suspension Design}

In this section a realistic vehicle suspension design is studied to maximize the enterprise level profit objective. The market consists of twelve sedans.

\section{Medium Size Vehicle Demand Model}

A demand model is created to analyze the effect of incremental engineering design changes on the market share of the vehicle ${ }^{19}$ (see Figure 9). Discrete Choice Analysis (DCA) models can be used to model the decision making behavior of individuals that choose one alternative from a finite set of mutually exclusive and collectively exhaustive alternatives. A key concept in DCA is the use of random utility to address unobserved taste variations, unobserved attributes, and model deficiencies. Random utility entails the assumption that the individual's true utility $U_{i n}$ consists of a deterministic or observable part $W_{i n}$ and a random unobservable disturbance $\epsilon_{i n}$.

$$
U_{i n}=W_{i n}+\epsilon_{i n}
$$

A quantitative process based on multinomial analysis is used to generate the demand model. The deterministic part of the utility can be parameterized as a function of observable independent variables (key product attributes $\mathbf{x}$, socioeconomic and demographic attributes $\mathbf{x}_{\mathbf{e}}$,

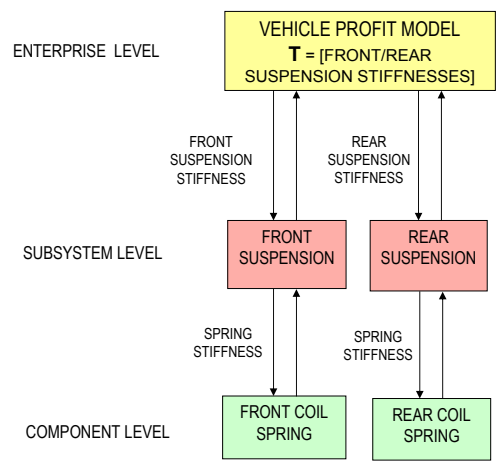

Figure 9. Schematic of vehicle profit and suspension design model. and price $P$ ) and unknown coefficients $\beta$, which can be estimated by observing the choices respondents make and thus represent the respondents' preference. The $\beta$ coefficients and utility functions are indicated with the subscript $n$, representing the $n^{\text {th }}$ respondent, the index $i$ refers to the $i^{\text {th }}$ choice alternative. There is no functional form imposed on the utility function $W$, i.e., $W$ can be additive, multiplicative, quadratic, etc. However, for the purposes of this paper, an additive form of the utility function, linear in the $\beta$ coefficients is used.

$$
W_{i n}=f\left(\mathbf{x}_{i}, P_{i}, \mathbf{x}_{\mathbf{e}, n}: \beta_{n}\right)
$$


The demand model $Q$ makes predictions on the change in market share, i.e., change in the number of vehicles sold of a particular make. Enterprise level utility is defined as the total profit, Eq. (13). Price of a vehicle $P$ is a constant and $C_{\text {susp }}$ and $C_{0}$ represent the cost for the suspension subsystems and for the rest of the vehicle system, respectively. Based on vehicle quality survey data from J.D. Power and Associates, the coefficients for the demand model are obtained using MNL as shown in Table 3, and the suspension cost is assumed to be linearly proportional to the suspension stiffnesses. For the current study values for a domestic sedan with $P=20,000, C_{0}=18,100, a_{f}=0.05$ and $a_{r}=0.05$, are used.

$$
\begin{aligned}
\Pi & =Q\left(P-C_{\text {susp }}-C_{0}\right) \\
& =Q\left(k_{s f}, k_{s r}\right)\left(P-a_{f} k_{s f}-a_{r} k_{s r}-C_{0}\right)
\end{aligned}
$$

Starting with a baseline specification that includes alternative specific constants (ASC), i.e., essential product attributes and demographic variables, the demand model is improved incrementally by adding additional attributes to the model. Alternative specific constants are added to represent the average preference of individuals for an alternative relative to a reference alternative and also to account for the average effect of all explanatory variables. The estimated models are then evaluated on several criteria including behavioral realism or their ability to model customer behavior in line with the analyst's expectations and goodness of empirical fit to the data. The coefficients in the model should have signs and magnitudes that are consistent with our understanding. For example, one would expect that the coefficient for the explanatory variable related to fuel economy to be positive, i.e., one would typically expect more cars to be sold when there is an improvement in fuel economy. Similarly, one would generally expect a dip in market share when the retail price of the car is hiked. Current demand model indicates that softer front suspension and stiffer rear suspension lead to the most profit for the market (Figure 10). In Figure 10 the shaded regions indicate a disconnected feasible domain for the suspension design.

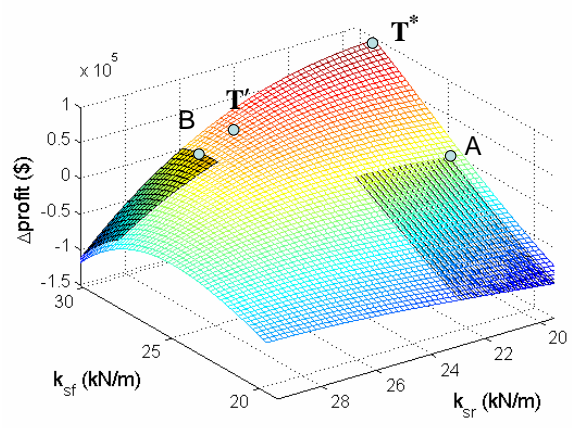

Figure 10. Vehicle demand model: profit change with respect to suspension stiffness changes. The shaded areas represent feasible suspension design domain. The baseline vehicle suspension stiffnesses are $k_{s f}=25.5(\mathrm{kN} / \mathrm{m})$ and $k_{s r}=19.5(\mathrm{kN} / \mathbf{m})$.

The statistical goodness of fit is evaluated using likelihood estimates and pseudo $R$-square values, a performance measure evaluated with the equally likely or zero model and the constants only model as reference model. The equally likely model is a model that has no parameters, i.e., the individual is assumed to have equal probability of choosing any of the alternatives in the choice set available to him. The constants only model includes only a full set of constants, i.e., constants corresponding to each of the alternatives with one of the alternatives chosen as the base alternative, as explanatory variables. The model estimation is carried out by maximizing the log likelihood using STATA. ${ }^{25}$

$$
\begin{aligned}
& \rho_{o}=1-\frac{L L(\text { model })}{L L(0)} \\
& \rho_{c}=1-\frac{L L(\text { model })}{L L(c)}
\end{aligned}
$$

$L L(0)$ and $L L(c)$ are the log likelihood estimates of the zero and constants only models.

\section{Implementation}

The demand model developed is used to assess the impact of suspension design changes on vehicle profit (see Figure 9). Twelve vehicles (seven models and twelve trims) are considered in the demand model representing the midsize car segment. Considering other segments, for example, sports models or pick-up trucks, would run the risk of yielding models that have a heavy demographic bias and are therefore not very sensitive to changes in product attributes. The assumption is that customers only consider vehicles from the midsize car segment, and specifically the twelve vehicle trims, when making their decision. While modeling suspension characteristics, a number of product attributes including retail price, resale value, annual percentage rate (APR) for auto loan, fuel economy, vehicle dependability index (VDI: a quality measure, expressed in terms of defects per 100 parts), vehicle length, and demographic attributes like customer age and income are included. Note 
that demographic attributes can only be included as alternative specific variables ${ }^{9}$ due to the nature of the MNL. Here 11 income variables corresponding to alternatives 2 to 12 are used and they are evaluated with alternative 1 as a reference. Similarly, the age variable is assumed to be equal for all domestic cars and is evaluated with respect to imported cars. The number of survey correspondents was 3881 . Front and rear suspension spring stiffnesses are used to model suspension characteristics.

Table 3 includes results of the model estimation and several observations can be made. Negative signs

Table 3. Results of Demand Model Estimation

\begin{tabular}{|c|c|c|c|c|}
\hline Variable type & Description & $\beta$ Coefficient & t-value & $95 \%$ Confidence interval \\
\hline \multirow{5}{*}{ Demographic } & Income 2 & 0.13 & 6.41 & $(0.09,0.18)$ \\
& Income 3 & 0.01 & 0.48 & $(-0.03,0.05)$ \\
& Income 4 & 0.06 & 2.57 & $(0.01,0.11)$ \\
& Income 5 & -0.10 & -4.2 & $(-0.15,-0.05)$ \\
& Income 6 & -0.08 & -3.37 & $(-0.13,-0.03)$ \\
& Income 7 & 0.07 & 2.99 & $(0.02,0.11)$ \\
& Income 8 & 0.08 & 3.22 & $(0.03,0.12)$ \\
& Income 9 & 0.08 & 3.25 & $(0.03,0.14)$ \\
& Income 10 & 0.19 & 9.38 & $(0.15,0.23)$ \\
& Income 11 & 0.05 & 2.29 & $(0.01,0.10)$ \\
& Income 12 & 0.04 & 1.18 & $(-0.02,0.10)$ \\
\hline Demographic & Age & 0.13 & 12.37 & $(0.11,0.15)$ \\
& Retail Price & -1.57 & -4.14 & $(-2.31,-0.82)$ \\
& Resale Value & 2.15 & 2.54 & $(0.49,3.80)$ \\
& Vehicle Dependability Index & -1.69 & -1.49 & $(-3.92,0.53)$ \\
& Annual Percentage Rate & -1.05 & -1.34 & $(-2.58,0.49)$ \\
& Fuel Economy & 0.64 & 1.51 & $(-0.19,1.46)$ \\
& Vehicle Length & -0.60 & -0.5 & $(-2.95,1.74)$ \\
& Front Suspension Stiffness & 1.75 & 3.11 & $(0.65,2.85)$ \\
& Rear Suspension Stiffness & 0.88 & 1.28 & $(-0.47,2.24)$ \\
\hline
\end{tabular}

Table 4. Iteration History: Maximizing Profit with Vehicle Suspension Design Change

\begin{tabular}{r|c|c|c||c|c|c}
\hline Iteration & $\begin{array}{c}\text { Target } \\
k_{s f}(\mathrm{kN} / \mathrm{m})\end{array}$ & $\begin{array}{c}\text { Target } \\
k_{s r}(\mathrm{kN} / \mathrm{m})\end{array}$ & $\begin{array}{c}\text { Desired } \\
\text { utility }(\$)\end{array}$ & $\begin{array}{c}\text { Response } \\
R_{1}\end{array}$ & $\begin{array}{c}\text { Response } \\
R_{2}\end{array}$ & $\begin{array}{c}\text { Actual } \\
\text { utility }(\$)\end{array}$ \\
\hline 1 & 30.2 & 19.5 & 80460 & 25.0 & 19.5 & -8935 \\
2 & 28.6 & 24.5 & 33701 & 29.4 & 25.0 & 27234 \\
\hline
\end{tabular}

of retail price, VDI, APR and vehicle length mean that customers prefer lower values for these variables, i.e., customers prefer cheaper cars, lower interest rates, fewer defects and cars that facilitate easy parking. Positive sign for fuel economy means that customers prefer higher gas mileage and positive signs for the suspension stiffnesses mean that stiffer suspensions are preferred. A stiffer suspension generally translates to better handling and load-carrying abilities but also results in a harsher ride. In this context, the current choice model indicates that customers value handling characteristics more than ride quality. Also, since we are dealing with variables normalized with respect to their extreme values, the magnitude of the coefficients should reflect their relative importance.

Table 4 summarizes the iteration process for the algorithm. The utopia target for suspension design is given at $\mathbf{T}^{*}=(30.2,19.5)(\mathrm{kN} / \mathrm{m})$ with profit $\$ 80,460$ corresponding to the peak point on the utility surface in Figure 10. Due to engineering feasibility the design with the minimum deviation from the utopia target is found at point $\mathbf{A}=(25.0,19.5)(\mathrm{kN} / \mathrm{m})$ with $-\$ 8,935$ profit (i.e., loss). Based on this design, the algorithm imposes a limiting constraint (Eq. (4)) to the enterprise problem. The modified enterprise problem finds a new optimal target with the maximum utility at $\mathbf{T}^{\prime}=(28.6,24.5)(\mathrm{kN} / \mathrm{m})$ with $\$ 33,701$ profit. This new 
target guides the ATC process to reach $\mathbf{B}=(29.4,25.0)(\mathrm{kN} / \mathrm{m})$ with the improved profit $\$ 27,234$. Note that the utopia targets assigned to the ATC problem are not achievable. However, the algorithm guides the iteration process to a design where the best available utility is obtained. Detailed suspension and coil spring designs by ATC are summarized in Table $5-8$.

\section{Conclusions}

Enterprise product planning has been systematically linked to the product development process. The proposed algorithm performed successfully in the examples. It is common not to achieve aggressive product performance targets perfectly, and the proposed algorithm allows design exploration within the capability of the product development teams often represented with a disconnected feasible domain. When the product design feasible domain is disconnected due to the discrete nature of the product components or manufacturing limitations with the OEMs, the proposed algorithm systematically assigns different targets for the engineering teams to meet the enterprise objective better. Analytical target cascading was successfully incorporated to achieve the performance targets with the minimum deviations while maximizing the utility at the enterprise level with the guidance of the proposed algorithm.

Future work involves adopting the nested multinomial logit model that can be more effectively incorporated in the multilevel decision making scenarios as well as in heterogeneous market segments (e.g., considering sedans with sport utility vehicles). Also, the sensitivity of product attributes to profit from the enterprise model can be utilized in the engineering design model.

\section{Appendix}

Table 5. Front Suspension Design

\begin{tabular}{|l|c|c|c|}
\hline Front suspension subsystem design & Optimal value & Lower bound & Upper bound \\
\hline Linear coil spring stiffness $(\mathrm{N} / \mathrm{mm})$ & 113.4 & 30 & 160 \\
Spring free length (mm) & 375.1 & 300 & 650 \\
Torsional stiffness (N-m/deg) & 30 & 20 & 85 \\
Overall front suspension stiffness (N/mm) & 29.3 & 19 & 30.2 \\
Suspension travel (m) & 0.099 & 0.05 & 0.1 \\
\hline
\end{tabular}

Table 6. Rear Suspension Design

\begin{tabular}{|l|c|c|c|}
\hline Rear suspension subsystem design & Optimal value & Lower bound & Upper bound \\
\hline Linear coil spring stiffness $(\mathrm{N} / \mathrm{mm})$ & 70.5 & 30 & 160 \\
Spring free length (mm) & 472.4 & 300 & 650 \\
Torsional stiffness (N-m/deg) & 69.3 & 20 & 85 \\
Overall rear suspension stiffness (N/mm) & 25 & 19 & 30.2 \\
Suspension travel (m) & 0.099 & 0.05 & 0.1 \\
\hline
\end{tabular}

Table 7. Front Coil Spring Design

\begin{tabular}{|l|c|c|c|}
\hline Front coil spring component design & Optimal value & Lower bound & Upper bound \\
\hline Wire diameter $(\mathrm{m})$ & 0.015 & 0.005 & 0.03 \\
Coil diameter $(\mathrm{m})$ & 0.077 & 0.05 & 0.2 \\
Pitch & 0.04 & 0.04 & 0.1 \\
Linear coil spring stiffness (N/mm) & 114.3 & & \\
Spring bending stiffness (N-m/deg) & 28.5 & & \\
\hline
\end{tabular}


Table 8. Rear Coil Spring Design

\begin{tabular}{|l|c|c|c|}
\hline Rear coil spring component design & Optimal value & Lower bound & Upper bound \\
\hline Wire diameter $(\mathrm{m})$ & 0.02 & 0.005 & 0.03 \\
Coil diameter $(\mathrm{m})$ & 0.154 & 0.05 & 0.2 \\
Pitch & 0.05 & 0.05 & 0.1 \\
Linear coil spring stiffness (N/mm) & 73.4 & & \\
Spring bending stiffness (N-m/deg) & 59.3 & & \\
\hline
\end{tabular}

\section{Acknowledgment}

This work has been partially supported by the NSF (DMII Grant 0335880) and the US Army TACOM through the Automotive Research Center at the University of Michigan and the Dual Use Science and Technology Program. This support is gratefully acknowledged. J.D. Power \& Associates is also acknowledged for providing vehicle quality survey data. The authors appreciate helpful suggestions by Frank Koppelman, Henk Jan Wassenaar and Geoff Rideout. The views presented here are those of the authors and do not necessarily reflect the views of others.

\section{References}

${ }^{1}$ Donndelinger, J. and Cook, H., "Methods for Analyzing the Value of Vehicles," Society of Automotive Engineers, February 1997.

${ }^{2}$ Besharati, B., Azarm, S., and Farhang-Mehr, A., "On the Aggregation of Preferences in Engineering Design," Proceedings of DETC 2001 ASME Design Engineering Technical Conference, Pittsburgh,PA, September 2001.

${ }^{3}$ Besharati, B., Azarm, S., and Farhang-Mehr, A., "A Customer-based Expected Utility metric for Product Design Selection," Proceedings of DETC 2002 ASME Design EngineeringTechnical Conference, Montreal, Canada, October 2002, DETC2002/DAC-34081.

${ }^{4} \mathrm{Li}, \mathrm{H}$. and Azarm, S., "Product Design Selection under Uncertainty and with Competitive Advantage," Proceedings of DETC 2000 ASME Design Engineering Technical Conference, Baltimore, MD, September 2000, DETC2000/DAC-14234.

${ }^{5}$ Wassenaar, H. and Chen, W., "An Approach to Decision Based Design with Discrete Choice Analysis for Demand Modeling," Transactions of ASME: Journal of Mechanical Design, Vol. 125, No. 3, 2003, pp. 490497.

${ }^{6}$ Cooper, A. B., Georgiopoulos, P., Kim, H. M., and Papalambros, P. Y., "Analytical Target Setting: An Enterprise Context in Optimal Product Design," Proceedings of the 2003 ASME Design Automation Conference, Chicago, IL, September 2003, DAC-48734.

${ }^{7} \mathrm{Gu}, \mathrm{X}$., Renaud, J., Ashe, L., Batill, S., Budhiraja, A., and Krajewski, L., "Decision-Based Collaborative Optimization," Transactions of ASME: Journal of Mechanical Design, Vol. 124, No. 1, 2002, pp. 1-13.

${ }^{8}$ Michalek, J., Feinberg, F., and Papalambros, P., "Linking Marketing and Engineering Product Design Decisions via Analytical Target Cascading," Journal of Product Innovation Management: Special Issue on Design and Marketing in New Product Development, 2004, to appear.

${ }^{9}$ Ben-Akiva, M. and Lerman, S., Discrete Choice Analysis, The MIT Press, Cambridge, MA, 1985.

${ }^{10}$ Hazelrigg, G., Systems Engineering: An Approach to Information-Based Design, Prentice-Hall, Inc., Upper Saddle River, NJ, 1996.

${ }^{11}$ Thurston, D., "Real and Misconceived Limitations to Decision Based Design with Utility Analysis," Transactions of ASME: Journal of Mechanical Design, Vol. 123, No. 2, 2001, pp. 176-182.

${ }^{12}$ Wassenaar, H. J., Chen, W., Cheng, J., and Sudjianto, A., "Enhancing Discrete Choice Demand Modeling for Decision-Based Design," Proceedings of DETC 2003 ASME Design EngineeringTechnical Conference, Chicago,IL, September 2003, DETC2003/DTM-48683.

${ }^{13}$ Wassenaar, H. J., Chen, W., Cheng, J., and Sudjianto, A., "An Integrated Latent Variable Choice Modeling Approach to Enhancing Product Demand Modeling," Proceedings of DETC 2004 ASME Design EngineeringTechnical Conference, Salt Lake City, UT, 2004, DETC2004-57487. 
${ }^{14}$ Sobieszczanski-Sobieski, J. and Haftka, R., "Multidisciplinary Aerospace Design Optimization Survey of Recent Developments," Structural Optimization, Vol. 14, No. 1, 1997, pp. 1-23.

${ }^{15}$ Kroo, I., "Miltidisciplinary Optimization Applications in Preliminary Design - Status and Directions," Proceedings of the 38th AIAA/ASME/ASCE/HS/ASC, Structures, Structural Dynamics and Materials Conference, Kissimmee, FL, September 1997, AIAA 97-1408.

${ }^{16}$ Michelena, N., Kim, H. M., and Papalambros, P. Y., "A System Partitioning and Optimization Approach to Target Cascading," Proceedings of the 12th Int. Conf. on Engineering Design, Vol. 24, Munich, Germany, 1999, pp. 1109-1112.

${ }^{17}$ Kim, H. M., Target Cascading in Optimal System Design, Ph.D. thesis, The University of Michigan, Ann Arbor, MI, 2001.

${ }^{18}$ Kim, H. M., Michelena, N. F., and Papalambros, P. Y., "Target Cascading in Optimal System Design," Transactions of ASME: Journal of Mechanical Design, Vol. 125, No. 3, 2003, pp. 474-480.

${ }^{19}$ Kim, H. M., Rideout, D. G., Papalambros, P. Y., and Stein, J. L., "Analytical Target Cascading in Automotive Vehicle Design," Transactions of ASME: Journal of Mechanical Design, Vol. 125, No. 3, 2003, pp. 481-489.

${ }^{20}$ Sobieszczanski-Sobieski, J., "Optimization by Decomposition: A Step from Hierarchic to Nonhierarchic Systems," Tech. Rep. NASA CP-3031, NASA Langley Research Center, Hampton, VA, 1988.

${ }^{21}$ Braun, R., Collaborative optimization: An architecture for large-scale distributed design, Ph.D. thesis, Stanford University, Stanford, CA, 1996.

${ }^{22}$ Sobieszczanski-Sobieski, J., Agte, J., and Sandusky, R., "Bi-Level Integrated System Synthesis (BLISS)," Technical Memorandum TM-1998-208715, NASA Langley Research Center, Hampton, VA, 1998.

${ }^{23}$ Green, P. and Srinivasan, V., "Conjoint Analysis Marketing: New Developments with Implications Research and Practice," Journal of Marketing, 1990.

${ }^{24}$ Kooppelman, F. and Sethi, V., Handbook of Transport Modeling, Pergamon Press, 2000.

${ }^{25}$ STATA Corporation, Stata Programming Reference Manual, 2003, ISBN 1-881228-73-8. 